\title{
Infliximab en el tratamiento de la enfermedad de Crohn. Estudio preliminar
}

\author{
José G ellona $\mathrm{V}^{1 \mathrm{a}}$, Antón Zarraonandia $\mathrm{A}^{\text {la }}$, Alvaro Zúníga $\mathrm{D}^{2}$, \\ Renato Palma $\mathrm{C}^{3}$, Jaime Contreras $\mathrm{P}^{4}$, Jaime Silva $\mathrm{Ch}^{5}$, \\ Róbinson G onzález D ${ }^{6}$, Carlos Q uintana V ${ }^{6,7}$. \\ Infliximab in the treatment \\ of patients with Crohn's disease. \\ Preliminary report
}

Background: Tumor necrosis factor alpha, interleukin 6 and 1 have a pathogenic role in Crohn's disease. Infliximab, a monoclonal antibody against tumor necrosis factor, is useful in the treatment of Crohn's disease, specially when the disease is active and associated to fistula formation. Aim: To assess the effectiveness of Infliximab in patients with Crohn's disease. Material and methods: Twelve patients with Crohn's disease (aged 16 to 63 years, 10 women) refractory to treatment with steroid, 5-aminosalicylic acid, antimicrobials and immunomodulatory drugs, were studied. A single intravenous $5 \mathrm{mg} / \mathrm{kg}$ dose Infliximab was administered. The Crohn's disease activity index (CDAI) was measured before and one week after the administration of the drug. Results: CDAI before and after Infliximab administration was $357 \pm 62$ and $138 \pm 122$ score points, respectively $(p<0.005)$. According to this score, five patients had a severe and seven a moderate disease flare. The disease remitted in 7 (58\%) and a clinical response was observed in $75 \%$. Conclusions: A single dose of Infliximab was effective to induce remission of the disease in $75 \%$ of this selected group of patients with Crohn's disease (Rev Méd Chile 2006; 134: 320-5).

(Key words: Antibodies, monoclonal; Crohn's disease; Infliximab; Tumor necrosis factor-alpha)

\footnotetext{
Recibido el 30 de diciembre, 2004. Aceptado el 5 de septiembre, 2005.

${ }^{1}$ Facultad de Medicina Universidad de Los Andes. Santiago, Chile. ${ }^{2}$ Departamento de Cirugía Digestiva y ${ }^{6}$ Gastroenterología, Facultad de Medicina, Pontificia Universidad Católica de Chile. ${ }^{3}$ Departamento de Gastroenterología, Clínica las Condes. Santiago Chile. ${ }^{4}$ Departamento de Cirugía, Hospital Clínico San Borja Arriarán, Universidad de Chile. ${ }^{5}$ Hospital del Cobre, CODELCO. Calama, Chile. ${ }^{7}$ Departamento de Medicina Interna, Facultad de Medicina, Universidad de Los Andes, Santiago, Chile anterno de Medicina
}

$\overline{\text { Correspondencia a: Dr. Carlos Quintana V. Departamento de }}$ Gastroenterología, Facultad de Medicina, Pontificia Universidad Católica de Chile. Marcoleta 367, Casilla 114d, Santiago. Fono: 3543820. Fax: 6397780. E mail: cquintan@med.puc.cl 
$\mathrm{L}$ a enfermedad de Crohn (EC), es una de las enfermedades inflamatorias crónicas intestinales. Estas se caracterizan por una reacción inflamatoria exagerada que produce daño de la pared intestinal.

La EC se caracteriza por producir una inflamación transmural, que afecta cualquier parte del tubo digestivo, especialmente el íleon y colon. Su curso es crónico recurrente, con recaídas y remisiones.

Los mecanismos desencadenantes son complejos y consistirían en la interacción de al menos tres elementos: factores de susceptibilidad genética, interacción con la flora microbiana entérica en una mucosa con permeabilidad aumentada, y una respuesta inmune anómala que produce daño tisular. Ente los factores genéticos, la mutación puntual que más se ha asociado con EC es el gen NOD2, ubicado en el cromosoma 16q12. También se han descrito mutaciones en los cromosomas 5, 6, 12, 14 y 19. Todas estas alteraciones genéticas se relacionan con la modulación de la respuesta inmune ${ }^{1,2}$ o la mantención de la barrera mucosa en el intestino ${ }^{3}$. Si bien estos factores genéticos son importantes, la incidencia en gemelos monocigotos es menor al $50 \%$, lo que implica la presencia de factores ambientales predisponentes ${ }^{4}$. Entre ellos destaca el hecho de que una menor exposición a agentes patógenos entéricos, como ocurre en países desarrollados, se acompaña de una mayor prevalencia de esta enfermedad. Otro factor ambiental es el consumo de tabaco, el cual se ha asociado a una peor evolución clínica de la $\mathrm{EC}^{3}$. Los antiinflamatorios no esteroidales (AINE) también se han relacionado con la reactivación de la actividad de las enfermedades inflamatorias intestinales.

Normalmente, la mucosa expuesta a un antígeno bacteriano genera una respuesta inmune, principalmente de tipo humoral. En ella, la baja permeabilidad de la barrera mucosa permite que las células presentadoras de antígenos (APC) estimulen una reacción de los linfocitos Th2 y Th3, causando la liberación de interleuquina (IL)-4, IL-5, IL-10 y TGF beta, que estimulan linfocitos $\mathrm{B}$, los que producen $\operatorname{IgA}^{5}$. En la EC la permeabilidad de la mucosa está alterada, presentándose en forma directa los antígenos contenidos en el lumen intestinal a los macrófagos y monocitos, los que liberan IL-12, induciendo una respuesta inmune celular (Th1). Esta respuesta consiste en la liberación de grandes cantidades de factor de necrosis tumoral-alfa (TNF-alfa), IL-6 e IL-
12 que inhiben la apoptosis de monocitos, macrófagos y linfocitos $\mathrm{T}$, causando inflamación de la pared intestinal y perpetuación de la respuesta inmune, entre otros efectos.

A partir de la demostración de estos mecanismos fisiopatológicos de la EC hasta ahora conocidos, han surgido nuevas posibilidades de tratamiento. Una de las líneas terapéuticas consiste en el bloqueo, mediante anticuerpos monoclonales, de las citoquinas involucradas. El infliximab es un anticuerpo monoclonal quimérico, híbrido (humano-ratón) anti TNF-alfa del tipo $\operatorname{IgG} 1^{6}$. Los mecanismos de acción de esta droga no están del todo claros, pero se plantea que se une al TNF-alfa soluble y también al TNF-alfa unido a la membrana celular de monocitos y macrófagos, induciendo su apoptosis, reduciendo la angiogénesis, el reclutamiento de células inflamatorias y la cascada de citoquinas, bloqueando así la respuesta inmune $e^{7,8}$. Se ha demostrado clínicamente mejor respuesta en pacientes con EC activa de grado moderada-grave, refractaria o con respuesta insuficiente al tratamiento convencional (sulfasalazina, mesalazina, corticoesteroides, antibióticos e inmunosupresores), y también en EC fistulizada9. Siendo éstas las indicaciones principales para iniciar el tratamiento con esta droga.

Actualmente, el infliximab es la única droga comprobada en un estudio control de doble ciego que ha sido capaz de cerrar completamente las fístulas perianales ${ }^{10}$. Según Steinhart, la respuesta de pacientes con EC refractaria a tratamiento previo fue de $81 \%$, teniendo remisión completa el $48 \%{ }^{11}$. También se demostró que $86 \%$ de los pacientes con fístulas perianales responden a la primera dosis de esta droga ${ }^{12}$.

El propósito de este trabajo clínico es evaluar la eficacia del infliximab pacientes con EC, en nuestro medio.

\section{Materiales y MÉtodos}

Pacientes. Se estudió en forma retrospectiva la respuesta clínica de 12 pacientes con EC refractaria a tratamiento médico que cumplían los criterios para iniciar tratamiento con infliximab.

Método. Para objetivar la eficacia del anticuerpo, se utilizó el índice de actividad de la enfermedad de Crohn (CDAI) ${ }^{13}$ previo a la infusión de infliximab. Posteriormente se evaluó a los mismos pacientes, 
una semana después de la infusión de esta droga. El CDAI se puede apreciar en la Tabla 1. La dosis usada fue de $5 \mathrm{mg} / \mathrm{Kg}$, por vía intravenosa, en pacientes hospitalizados.

Se definió como respuesta clínica la disminución del puntaje de CDAI mayor o igual a 70 puntos o una reducción mayor o igual al 25\% del valor previo ${ }^{14}$. Además se consideró remisión de la enfermedad a un puntaje menor a 150 .

Criterios de inclusión. Se incluyó pacientes con EC grave, lo que definimos como CDAI mayor a 220 puntos, sin respuesta al tratamiento médico (corticosteroides, 5-aminosalicilatos, antibióticos, e inmunomoduladores como 6-mercaptopurina y azatioprina). También se incluyó a pacientes con enfermedad perianal. Se excluyeron aquellos pacientes que tenían enfermedad estenosante $u$ obstrucción intestinal secundaria a EC.

Estadística. Para poder comparar los promedios de puntaje de CDAI obtenidos, se aplicó la prueba t para dos promedios, con nivel de significación de $95 \%(\alpha<0,05)$

\section{Resultados}

Pacientes. Los pacientes fueron 2 hombres y 10 mujeres, entre 16 y 63 años, con un promedio de edad de $38 \pm 14$ años. En la Tabla 2 se describen las características individuales de cada paciente. Los segmentos del tubo digestivo más comprometidos fueron colon, principalmente como pancolitis, compromiso del colon descendente y recto. También se observó una alta frecuencia de compromiso perianal (Tabla 2). Dos de nuestros enfermos presentaron compromiso inflamatorio no estenosante del intestino delgado. De las complicaciones observadas previas a la infusión de la droga, 66\% de los pacientes presentaba fístulas, 33\% artralgias, 16\% tenía abscesos perianales y, también en 16\% se observó eritema nodoso. Otras complicaciones observadas con menor frecuencia fueron uveítis, epiescleritis $y$ estomatitis aftosa.

Evaluación de la respuesta. El CDAI promedio, previo a la administración de infliximab fue de $357 \pm 62$ puntos, una semana después fue de $138 \pm 122$ puntos ( $\mathrm{p}<0,005)$. En la Figura 1 se aprecia la respuesta de cada paciente al tratamiento. De acuerdo al CDAI, 5 pacientes (42\%) sufrían crisis grave y 7 pacientes (58\%) crisis moderada previo a la administración de la droga. Hubo remisión de la enfermedad en 7 pacientes (58\%), 3 pacientes (25\%) evolucionaron a actividad leve, 1 paciente mejoró parcialmente de actividad grave a moderada y 1 permaneció en crisis grave. Por lo tanto, según los criterios usados, se encontró respuesta clínica en 9 (75\%) pacientes.

Tabla 1. Índice de actividad de la EC (CD AI)13

\begin{tabular}{|c|c|c|}
\hline Variable & Descripción & Amplificador \\
\hline 1 & Número de deposiciones líquidas (cada día por 7 días) & $\mathrm{x} 2$ \\
\hline 2 & Dolor abdominal, suma de 7 evaluaciones diarias $(0=$ ninguno, 1 leve, $2=$ moderado, $3=$ intenso $)$ & $\mathrm{x} 5$ \\
\hline 3 & Estado general, suma de 7 diario $(0=$ bueno, $1,2,3=$ intermedio, $4=$ malo $)$ & $\mathrm{x} 7$ \\
\hline 4 & $\begin{array}{l}\mathrm{N}^{\circ} \text { de las siguientes complicaciones (artritis o artralgia, iritis o uveítis, eritema nodoso, } \\
\text { pioderma gangrenoso o estomatitis aftosa, fisura o fístula anal, fístula en otra localización, } \\
\text { fiebre superior a } 37,8^{\circ} \mathrm{C} \text { en la semana previa }\end{array}$ & $\mathrm{x} 20$ \\
\hline 5 & Uso de difenoxilato o loperamida para control de diarrea $(0=$ no, $1=$ sí $)$ & x30 \\
\hline 6 & Masa abdominal $(0=$ no, $2=$ dudosa, $5=$ presente $)$ & $\mathrm{x} 10$ \\
\hline 7 & Diferencia de hematocrito con respecto al normal ( $47 \%$ hombres, $42 \%$ mujeres) & $\mathrm{x} 6$ \\
\hline 8 & Porcentaje desviación del IMC standard & $\mathrm{x} 1$ \\
\hline
\end{tabular}

CDAI <150: Remisión

CDAI 150-250: Crisis Leve

CDAI 250-350: Crisis Moderado

CDAI >350: Crisis Grave

Respuesta clínica: Disminución del CDAI $\geq 70$ puntos o $\geq 25 \%$ del puntaje previo 
Tabla 2. Resumen de las características individuales de cada paciente

\begin{tabular}{|c|c|c|c|c|c|c|}
\hline Sexo & $\begin{array}{l}\text { Edad } \\
\text { (años) }\end{array}$ & $\begin{array}{l}\text { Años } \\
\text { EC }\end{array}$ & $\begin{array}{l}\text { Segmentos } \\
\text { comprometidos }\end{array}$ & $\begin{array}{l}\text { Complicaciones } \\
\text { tratamiento }\end{array}$ & $\begin{array}{l}\text { Evolución post } \\
\text { adversas }\end{array}$ & Reacciones \\
\hline $\mathrm{F}$ & 31 & 2 & Pancolitis, ano & $\begin{array}{l}\text { Reservoritis, eritema } \\
\text { nodoso, fístula } \\
\text { reservorio - perianal }\end{array}$ & Remisión & No \\
\hline $\mathrm{F}$ & 28 & 4 & $\begin{array}{l}\text { Colon Izq. ano, } \\
\text { recto }\end{array}$ & $\begin{array}{l}\text { Fisuras anales } \\
\text { cicatrización de } \\
\text { fisuras }\end{array}$ & Remisión, & No \\
\hline $\mathrm{F}$ & 38 & 6 & $\begin{array}{l}\text { Pancolitis, ano, } \\
\text { recto }\end{array}$ & Fístula recto-vaginal & Remisión, CPF* & No \\
\hline $\mathrm{F}$ & 23 & 8 & $\begin{array}{l}\text { Colon izq. } \\
\text { sigmoides }\end{array}$ & $\begin{array}{l}\text { Fístulas (3), fisura anal, } \\
\text { absceso perianal }\end{array}$ & $\begin{array}{l}\text { Crisis leve, } \\
\text { CPF* }\end{array}$ & No \\
\hline M & 49 & 6 & $\begin{array}{l}\text { ciego, } \\
\text { sigmoides, } \\
\text { recto }\end{array}$ & $\begin{array}{l}\text { Absceso perianal, fisura } \\
\text { anal, artralgias }\end{array}$ & Remisión & $\begin{array}{l}\text { Herpes } \\
\text { faríngeo }\end{array}$ \\
\hline M & 63 & 7 & Pancolitis & $\begin{array}{l}\text { Fisura anal, estomatitis } \\
\text { aftosa, episcleritis }\end{array}$ & Crisis leve & No \\
\hline $\mathrm{F}$ & 61 & 23 & Pancolitis, ano & $\begin{array}{l}\text { Estomatitis aftosa, } \\
\text { eritema nodoso, } \\
\text { artralgias, fiebre, } \\
\text { fístulas }\end{array}$ & Remisión, CPF* & No \\
\hline $\mathrm{F}$ & 30 & $?$ & Colon izq. recto & Fístula & $\begin{array}{l}\text { Crisis leve, } \\
\text { CPF* }^{*}\end{array}$ & $\begin{array}{l}\text { Artritis, } \\
\text { hepatitis }\end{array}$ \\
\hline $\mathrm{F}$ & 33 & 14 & Pancolitis & $\begin{array}{l}\text { Fístula recto-vaginal, } \\
\text { artralgias }\end{array}$ & $\begin{array}{l}\text { Crisis moderada, } \\
\text { CPF* }^{*}\end{array}$ & No \\
\hline $\mathrm{F}$ & 36 & 8 & Ano, recto & Fístulas (3) & $\begin{array}{l}\text { Crisis moderada, } \\
\text { CPF* }^{*}\end{array}$ & No \\
\hline F & 16 & 2 & Ileon terminal & - Remisión & No & \\
\hline $\mathrm{F}$ & 47 & 21 & $\begin{array}{l}\text { Ileon, Colon } \\
\text { izquierdo, ano }\end{array}$ & $\begin{array}{l}\text { Uveítis, eritema nodoso, } \\
\text { fístula, artritis }\end{array}$ & $\begin{array}{l}\text { Remisión } \\
\text { CPF* }^{*}\end{array}$ & No \\
\hline
\end{tabular}

${ }^{*} \mathrm{CPF}$ : Cierre parcial de fístulas.

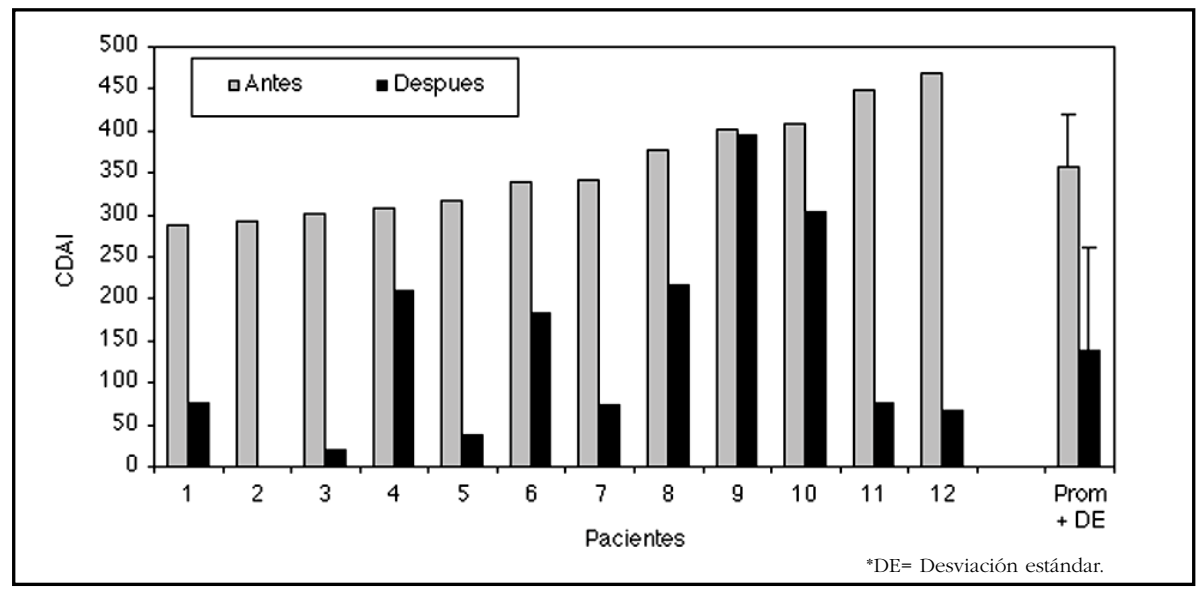

Figura 1. CDAI antes y después del tratamiento con Infliximab. 
Además de la respuesta clínica, en todos los pacientes que presentaban fístulas se observó una mejoría de éstas, que si bien no llegaron al cierre total, disminuyeron su tamaño y débito.

\section{DisCUSIÓN}

En 1997, Targan et $\mathrm{al}^{15}$, publicaron los resultados de un estudio controlado y aleatorio usando infliximab en pacientes con EC refractaria a otras terapias y puntaje de CDAI mayor a 220. Según este estudio, los pacientes tuvieron una respuesta inicial de 64\%. De acuerdo con nuestra observación, la primera dosis de infliximab ha logrado inducir respuesta clínica favorable en $75 \%$ y remisión en 58\% de nuestros pacientes, lo que concuerda con este y otros estudios publicados ${ }^{11,14,16}$. En nuestro trabajo sólo se evaluó el tratamiento en el corto plazo y no el observado con dosis de mantención, como es recomendado actualmente. Por consiguiente, no podemos comparar nuestros resultados con publicaciones que han descrito los resultados en un largo plazo. La terapia actual con infliximab recomendada es iniciar con dosis de $5 \mathrm{mg} / \mathrm{kg}$ en tres dosis aplicadas en la semana 0,2 y 6 . Luego, se recomienda mantener infusiones de 5 ó $10 \mathrm{mg} / \mathrm{kg}$ cada 8 semanas ${ }^{11,14}$. Nosotros evaluamos la respuesta a la primera dosis debido a que 9 de los 12 pacientes no pudieron seguir con la terapia recomendada por motivos económicos.

$\mathrm{Al}$ observar las características epidemiológicas y clínicas de la enfermedad en este grupo, no se logra encontrar algún factor que se relacione con la respuesta terapéutica observada, probablemente debido al escaso número de pacientes. La sola disminución del puntaje CDAI no ha significado necesariamente la mejoría total de las lesiones anatómicas. Creemos que ello se debe principalmente al corto período de observación; por ello, sería útil aumentar el número de pacientes y el tiempo de observación con el fin de confirmar y precisar estos resultados.

Sobre los efectos adversos registrados en los pacientes a quienes se les aplicó la droga, éstos se presentaron en 2 pacientes (16\%) (Tabla 2) y ninguno fue grave. Tampoco se presentaron efectos adversos secundarios a la infusión. Estos resultados, quizás se debieron a que el grupo estudiado fue pequeño y recibió una sola dosis de la droga. Colombel et $\mathrm{al}^{17}$ describieron $6 \%$ de reacciones adversas graves relacionadas con la infusión de infliximab en pacientes con EC. Dentro de estas reacciones graves destacan: infecciones graves, enfermedad del suero, reacción dérmica a la infusión, lupus inducido por drogas y desarrollo de tumores sólidos. El estudio ACCENT $\mathrm{I}^{14}$ menciona entre $3 \mathrm{y}$ $6 \%$ de reacciones adversas secundarias a la droga, siendo éstas cefalea, mareos, náuseas, irritación del sitio de punción de la infusión, eritema, dolor torácico, disnea y prurito. Reacciones adversas serias se describieron en 3\% del grupo de pacientes estudiados. Un punto que no podemos dejar de mencionar, es el aumento reportado de infecciones granulomatosas, especialmente tuberculosis (TBC), asociadas al uso de infliximab ${ }^{18}$. La frecuencia descrita es de aproximadamente 143 casos por 100.000 tratados $^{18}$, aparecen en su mayoría dentro de los primeros 90 días de tratamiento, principalmente como reactivación de TBC latente y que generalmente se manifiestan como enfermedad extrapulmonar o diseminada. Por esto, se recomienda la búsqueda de TBC mediante historia clínica y PPD, previo al inicio de la terapia con infliximab ${ }^{19}$ y mantener un alto índice de sospecha durante el tratamiento. Existe consenso que los pacientes con TBC activa no deberían ser tratados hasta la resolución del cuadro infeccioso. En pacientes con enfermedad latente, estaría indicada la terapia preventiva con isonizida (HIN) por 9 meses, pero no está claro si se debe esperar los 9 meses de HIN para iniciar la terapia con infliximab, o si se podría administrar asociado a la terapia preventiva ${ }^{19}$. Por ahora, esto quedaría a criterio del tratante, esperamos en el futuro tener guías aplicables a nuestro medio con respecto a este punto.

Rosenstiel et al describieron que el TNF-alfa aumentaría la expresión de ARNm producido por el gen NOD2 mutado $^{20}$, lo que se relaciona con mayor incidencia de EC. Debido a que no poseemos la técnica para identificar la mutación puntual del gen NOD2, no se pudo distinguir entre aquellos pacientes que poseían la mutación y aquellos que no la poseían. Hubiese sido interesante comparar la respuesta a la infusión de infliximab entre los pacientes con la mutación y aquellos sin ésta.

Un punto no evaluado objetivamente en este trabajo, por su carácter retrospectivo, ha sido la 
calidad de vida y el estado general de los pacientes luego del tratamiento. Este hecho no nos permite concluir sobre el estado subjetivo de ellos con posterioridad al tratamiento.

\section{REFERENCIAS}

1. Van Heel DA, McGovern DPB, Jewell DP. Crohn's disease: genetic susceptibility, bacteria, and innate immunity. Lancet 2001; 441: 1902-4.

2. Van Heel Da, Udalova I, De Silva A, Mc Govern D, KinOUChI Y, Hull J ET AL. Inflammatory bowel disease is associated with a TNF polymorphism that affects an interaction between the OCT1 and NF- $\kappa B$ transcription factors. Hum Mol Genet 2002; 11: 1281-9.

3. Shanahan F. Crohn's disease. Lancet 2002; 359: 62-9.

4. TaYlor KD, Rotter JI, Yang H. Genetics of inflammatory bowel disease. In: Targan SR, Shanahan F, Karp LC, eds. Inflammatory bowel disease: from bench to bedside. Dordrecht: Kluwer Academic Publishers (in press).

5. Gordon JL, Hooper LV, Mcnevin SM, Wong M, Bry L. Epithelial cell growth and differentiation III. Promoting diversity in the intestine: conversations between the microflora, epithelium, and diffuse GALT. Am J Physiol 1997; 273: 565-70.

6. Aguillón J, Contreras J, Dotte A, Cruzat A, Catalán D, Salazar L ET aL. Nuevas armas inmunológicas para la medicina del siglo XXI: Terapia biológica basada en el uso de anticuerpos monoclonales de última generación. Rev Méd Chile 2003; 131 : 1445-53.

7. Shanahan F. Probiotics and inflammatory bowel disease: is there a scientific rationale? Inflamm Bowel Dis 2000; 6: 107-15.

8. Parsi M, AchKar JP, Richardson S, Katz J, Hammel JP, Lashner BA et al. Predictors of response to infliximab in patients with Crohn's disease. Gastroenterology 2002; 128: 707-13.

9. Feldermann M, Maini RN. Anti-TNF alpha therapy of rheumatoid arthritis: what have we learned? Ann Rev Inmunol 2001; 19: 163-96.

10. Present DH, Rutgeers P, Targan S, Hanauer SB, Mayer L, Van Hogezand RA et aL. Infliximab for the treatment of fistulas in patients with Crohn's disease. N Engl J Med 1999; 340: 1398-405.
Si bien este trabajo es una aproximación inicial, preliminar, de corte transversal y sin seguimiento, creemos que puede tener utilidad, dado que proporciona datos sobre el tratamiento de la EC y sus resultados en nuestro medio.

11. Steinhart H. Maintenance therapy in Crohn's disease. Can J Gastroenterol(s) 2000; 14: 23-8.

12. Tapstad DR, Panaccione R, Heine JA. Combined set on placement, infliximab infusion, and maintenance immunosuppressives improve healing rate in fistulizing anorectal Crohn's disease: a single center experience. Dis Colon Rectum 2003; 46 : 577-83.

13. Sandborn W, Feagan B, Hanauer S, Lochs $H$, Lofberg R, Modigliani R et al. A review of activity indices and efficacy endpoints for clinical trials of medical therapy in adults with Crohn's disease. Gastroenterology 2002; 122: 512-30.

14. Hanauer S, Feanagan B, Mayer L, Schreiber S, Colombel JF, Rachmilewitz D et al. Maintenance infliximab for Crohn's disease: the ACCENT I randomized trial. Lancet 2002; 359: 1541-99.

15. Targan S, Hanauer S, Van Deventer S, Mayer L, Present D, Braakman T et al. A short term study of chimeric monoclonal antibody cA2 to tumor necrosis factor aaa for Crohn's disease. N Engl J Med 1997; 337: 1029-35.

16. Rutgers P, Feagan B, Lichtenstein G, Mayer L et al. Comparison of scheduled and episodic treatment strategies of Infliximab in Crohn's disease. Gastroenterology 2004; 126: 402-13.

17. Colombel JF, Loftus E, Tremaine W, Egan L, Harmsen WS, Schleck C ET AL. The safety profile of infliximab in patients with Crohn's disease: The Mayo Clinic experience in 500 patients. Gastroenterology 2004; 126: 19-31.

18. Wallis RS, Broder MS, Wong JY, Hanson ME, BEENHOuwer DO. Granulomatous infections diseases associated with tumor necrosis factor antagonists. CID 2004; 38: 1261-65.

19. Long R, Gardam M. Tumour necrosis factor-alfa inhibitors and the reactivation of latent tuberculosis infection. CMAJ 2003; 168: 1153-6.

20. Rosenstiel P, Fantini M, Brautigam K, Kuhbacher T, Waetzig GH, Seegert D et al. TNF-aaa and IFNyyy regulate the expression of the NOD2 (CARD15) gene in the human intestinal epithelial cells. Gastroenterology 2003; 124: 1001-9. 